\title{
VIOLENCIA EN LA RELACIÓN DE PAREJA: UN DESAFÍO EN LA PROMOCIÓN DE SALUD Y PREVENCIÓN DE LA ENFERMEDAD
}

Nilsa M. Burgos Ortiz'

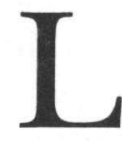

a violencia contra la mujer no es un fenómeno nuevo y menos aún, la violencia doméstica, aquella que ocurre al interior de la relación de pareja. Lo que es relativamente nuevo es la denuncia, el estudio y la intervención con esta violencia. A finales de la década del sesenta el movimiento feminista, especialmente en Estados Unidos, comenzó a hablar en voz alta sobre el maltrato conyugal, abriendo la puerta de la privacidad del hogar, a luchar porque se reconociera como problema social, a estudiarlo y combatirlo. De alguna manera, en la década de la mujer, particularmente en el 1975 -Año Internacional de la Mujer- en que se celebró la Primera conferencia Internacional de la Mujer en México, el mundo entero tuvo que hacer referencia a la existencia de esta violencia. Otras conferencias internacionales como la de Nairobi, Viena y Beijing le dieron continuidad a la discusión de la prevalencia de toda la violencia contra la mujer y presentaron una serie de acciones para su eliminación. De estas conferencias y el avance del movimiento de mujeres se han aprobado una serie de declaraciones y tratados que intentan erradicar la violencia y garantizar que las

\footnotetext{
${ }^{1}$ Catedrática, Escuela Graduada de Trabajo Social Beatriz Lassalle, Universidad de Puerto Rico.
} 
humanas también tenemos derechos humanos como es el de vivir en paz.

Mencionaré sólo dos de estos acuerdos. Primero tenemos la Declaración sobre la eliminación de la violencia contra la mujer firmada en 1993 por la Organización de las Naciones Unidas, que la reconoce como una violación a los derechos humanos y responsabiliza a los Estados para la implantación de medidas destinadas a la eliminación de la violencia de gériero. La otra medida firmada por la mayoría de los países miembros de la Organización de Estados Americanos (OEA) en 1994, es la Convención Interamericana para la prevención y sanción a la violencia en contra de las mujeres, que la reconoce como una ofensa en contra de la dignidad humana y como una manifestación de las históricas relaciones desiguales de poder entre los hombres y las mujeres (Comisión Interamericana de Mujeres, OEA, 1994). Como resultado de estos tratados y la activación política principalmente de mujeres, muchos de nuestros países cuentan por lo menos con una ley que ha servido de instrumento para reclamar justicia en contra de la violencia. En el caso de Puerto Rico es la Ley \#54 de agosto de 1989 - Para prevenir e intervenir con la violencia doméstica. En Panamá es la Ley \#27 del 16 de junio de 1995, por la cual se tipifican los delitos de violencia intra-familiar y maltrato de menores.

$\mathrm{La}$ violencia intra-familiar es un tema que concierne a toda la sociedad independientemente de grupo étnico o racial, condición económica, religión y nivel de escolaridad. La violencia en la relación de pareja es una de las manifestaciones de este tipo de violencia, a la que se le dará énfasis en este trabajo. Generalmente, las víctimas son mujeres de todas las edades; y los hombres son los victimarios, también de todas las edades. Sin embargo, la violencia no se puede circunscribir al interior de la familia pues esta es un reflejo de lo que ocurre en la sociedad. Se analizará cómo el proceso de socialización y formación de género; en que además de la familia, participan el sistema educativo, la religión y los medios de comunicación; también han propiciado la violencia contra las mujeres en los diferentes grupos de edad. Se discutirá también, la violencia en la relación de pareja como problema de salud pública y finalmente se presentarán algunas alternativas en la promoción de salud y prevención de la enfermedad. 


\section{Conceptos y perspectivas teóricas}

El maltrato conyugal se manifiesta de tres formas: físico, emocional o psicológico y abuso sexual. La violencia física se evidencia en golpes, quemaduras, cortaduras, mordidas, exposición a peligros, expulsión del hogar y negligencia. Es alarmante que vaya en aumento el número de mujeres que han perdido sus vidas a manos de sus esposos, ex-esposos, compañeros o ex-compañeros sentimentales. El Departamento de Justicia Criminal de Puerto Rico, señaló que entre 1996 al presente, 559 hombres fueron convictos por asesinatos $\mathrm{u}$ homicidios relacionados con la violencia en la relación de pareja. El derecho legal de un hombre a golpear a su esposa no está explícitamente reconocido en la mayoría de los países occidentales, pero el legado del patriarcado continúa generando las condiciones y relaciones que conducen a un marido a utilizar la fuerza contra la esposa. La idea de paz, seguridad y armonía todavía son muy asociados con la familia y por lo tanto, es difícil aceptar que existe violencia en la misma. Además, los intentos de encubrimiento en los que entran la mayoría de las mujeres victimizadas tienen un doble carácter, pues se trata tanto de convencer a los demás de que todo anda bien en la relación conyugal, de que no hay nada de problemático en ésta, como de persuadirse a sí mismas de que sus vidas no son el fracaso que sucesos de ese tipo podrían darles a entender que son (Silva Bonilla, 1990).

El otro tipo de violencia doméstica es la emocional o psicológica, que puede incluir burlas, insultos, amenazas, humillaciones, acusaciones y otras agresiones. Es una forma de violencia que por asumir expresiones muy sutiles puede, incluso, pasar desapercibida a nuestros niveles conscientes para ir a registrarse en las esferas inconscientes de nuestros procesos de conciencia (Silva Bonilla, 1985). Ciertamente, es un tipo de violencia difícil de observar y estudiar, pero a su vez el daño puede conducir a unos estados depresivos que podrían culminar en el suicidio.

El tercer tipo de violencia doméstica es el abuso sexual. Incluye la burla de la sexualidad de la mujer, la constante demanda del sexo con amenazas o a la fuerza, tocarla de una manera no grata para ellas, forzarla a desvestirse aún al frente de sus hijos(as) y hasta violarla. Es obvio que este tipo de violencia puede incluir maltrato físico y emocional lo que demuestra la dificultad de que puedan ocurrir en forma separada. Existe la tendencia a no considerar la 
violación en el matrimonio como tal, porque el derecho de la mujer «a decir que no" a su esposo es derogado por el "derecho" del hombre a hacer lo que quiera con su esposa. Sin embargo, esta experiencia puede resultar muy traumática, pues la víctima se queda viviendo con el victimario, y la violencia podrá repetirse porque no puede o se siente incapaz de denunciarlo.

$\mathrm{Al}$ analizar la violencia en la relación de pareja, es crucial entender el ciclo de la violencia, que se divide en tres fases. La primera fase, acumulación de tensión, se caracteriza por cambios repentinos en el ánimo del agresor, quien comienza a reaccionar negativamente ante lo que él siente como frustración de sus deseos. Pequeños episodios de violencia escalan hasta alcanzar el ataque mayor, pero son minimizados y justificados por ambas partes de la pareja. Los pequeños incidentes violentos se van manifestando con mayor frecuencia. En algún punto hacia el final de esta fase, es ya imposible controlar el proceso en marcha y se entra en la segunda fase. Esta es la descarga aguda de la violencia, la más corta de las tres, y consiste en la descarga incontrolada de las tensiones construidas durante la primera fase. El detonador para la explosión violenta puede ser lo mismo un hecho extremo, como por ejemplo el que ella haya salido sin avisar, o sólo el propio estado interno del agresor. Cuando pasa el ataque agudo le sigue un período inicial de "choque", negación del hecho, colapso emocional o sentimientos de desamparo. La última fase, luna de miel reconciliadora, es bienvenida por ambas partes de la pareja, pero irónicamente es el momento en que la victimización de la mujer se completa. En ésta, el agresor se conduce de un modo encantador; se siente mal por sus acciones y transmite su remordimiento. Promete a la mujer no volver a herirla e implora su perdón.

Existen varias teorías que aportan posibles explicaciones a la violencia familiar. Están las teorías socio-culturales, que demuestran cómo las normas culturales de disciplina permiten, en diferentes culturas, diferentes grados de violencia contra los niños(as) y que es probable que esta generación recurra a la violencia física como una manera de resolver los problemas. En otras palabras, plantean una relación entre maltrato de niños(as) y de la esposa. Las teorías psicológicas, principalmente el psicoanálisis, tienden a definir a la mujer agredida como masoquista, reforzando la culpa en estas mujeres y la subestimación de sí mismas. Los hombres son considerados muchas veces enfermos mentales, generalmente 
sicópatas que no son responsables de su comportamiento. Naturalmente esta teoría implicará que la mayoría de los hombres son enfermos mentales debido a la gran cantidad de agresores que existe.

La teoría de la comunicación plantea la existencia de un proceso inadecuado o disfuncional, de intercambio de información entre los miembros de la familia. Esta teoría afirma que la persona agresora utiliza la violencia para comunicarse, reafirmar su poder y expresar su disgusto. Otra teoría que se menciona desde principios de la década del ochenta es la de la desesperanza aprendida, que describe la forma en que algunas mujeres se ven atrapadas en relaciones abusivas. Walker (1980) plantea que la desesperanza aprendida es un estado de pasividad que resulta del proceso de victimización progresiva, en que una mujer se convence de que no existe forma de escaparse de su agresor, cree que su situación no tiene ninguna salida y por lo tanto no realiza ningún esfuerzo para que cambie.

Las teorías feministas plantean que toda violencia es reflejo de relaciones desiguales de poder, entre las cuales la más grande es entre hombres y mujeres. Los hombres, al asumir el rol de protectores de «sus mujeres», se sienten con el derecho de disciplinar y reprimir su propiedad. La mujer se ve a sí misma sin poder y atrapada en un sistema que da un doble mensaje: la violencia contra otra persona es ilegal y es castigada, excepto si es contra la esposa. La ideología patriarcal significa la manifestación e institucionalización del dominio de los hombres en la familia y la extensión de ese dominio sobre las mujeres en la familia y en la sociedad en general (Colectivo Ideologías y Vivencias de los Géneros, 1995). Esto no implica que las mujeres no tengan derechos y recursos, sino que en cada dimensión de la vida, tanto pública como privada, permea la idea y la práctica patriarcal. Cervantes Islas (2000), afirma que las expresiones autoritarias, antidemocráticas, sexistas, discriminatorias y demás prácticas que legitiman el poder masculino, permanecen invisibles e inmiscuidas en tantas instituciones, como en personas y manifestaciones culturales, y para muchos hombres son difíciles de detectar, pero esta situación privilegia lo masculino y a los hombres sobre lo femenino y sobre mujeres reales.

En una investigación reciente realizada en Puerto Rico sobre masculinidad y violencia en la relación de pareja, se encontró cómo en la construcción de las masculinidades, si bien comienza a ser objeto de cuestionamiento por los hombres, continúan vigentes los 
mandatos y referentes de la masculinidad hegemónica tradicional (Torres Hernández, 1999). En relación a la violencia en la relación de pareja, en esta investigación se observó que los hombres perciben los actos de maltrato cometidos por éstos como provocados por la agresión física o verbal de la pareja; y que en ocasiones, los hombres admiten haber provocado a su pareja para que esta los agredieran y de esta forma justificar como defensivas sus acciones. Otro estudio enfocó en las estrategias de enfrentamiento que utilizan las mujeres para lidiar con la violencia, clasificándolas en dos dimensiones: las estrategias dirigidas a resolver el problema alterando o cambiando el ambiente; y aquellas dirigidas a regular las emociones de la mujer (Valle Ferrer, 1998). Entre las estrategias emocionales están: distanciamientos, auto-control, búsqueda de apoyo social, escapar o evadir, asumir responsabilidad o culpa, y reapreciación positiva (Valle Ferrer, 1998).

Por otro lado, las reacciones de las mujeres que sufren maltrato son variadas y se complican a medida que permanece en esta violencia. Entre estas reacciones se encuentran: la ansiedad crónica, negación, vergüenza, culpa, impotencia, condiciones psicosomáticas, vulnerabilidad y comportamiento depresivo. No cabe duda que todas estas reacciones y la exposición cotidiana a la violencia implican un gasto de energía, tanto física como emocional, que pueden conducir al agotamiento e impiden que las mujeres se desarrollen plenamente. El cuerpo de conocimiento sobre el tema de la violencia en la relación de pareja está basado principalmente en investigaciones realizadas en Estados Unidos y Europa. Ellsberg, et al. (1998) nos aclaran que estudios en otros países tales como India, Bangladesh y Cambodia, indican que, aunque existen diferentes formas de justificar y practicar la violencia, las descripciones de las vivencias de las mujeres que experimentan la violencia: la vergüenza, el miedo, la justificación y auto-culpabilidad, la impotencia, son sorprendentemente parecidas; lo que implica que estas experiencias trascienden a las culturas específicas en que se desarrollan.

Entendiendo que la vida en violencia no es agradable, la pregunta que surge siempre es: ¿Por qué permanecen las mujeres en una situación de agresión? Una de las razones principales es la dependencia económica de las que no trabajan asalariadamente, las que trabajan por un salario inferior al del esposo o compañero sexual, o si las condiciones de empleo son muy precarias, enfrentando costos de vida muy altos. Otra razón es la familia, de tanta 
importancia en la cultura latinoamericana; y que aunque la mujer sabe que no puede vivir con un compañero violento, también tiene la responsabilidad de mantener la familia unida y evitarles el dolor de una separación. Existen otras presiones como son las ejercidas por las amistades y las religiosas, en particular el valor que se le asigna a perdonar a quienes nos agreden. $\mathrm{El}$ amor que las mujeres sienten por el hombre que les ha jurado amor toda la vida, las lleva muchas veces a creer que él cambiará y no la volverá a agredir. La dependencia emocional es otra de las razones para permanecer en una relación de violencia, que se manifiesta en el temor a la soledad y a no tener un hombre que las "proteja".

\section{Contexto socio-cultural}

La familia es una de las instituciones más importantes en América Latina, siendo primordial en el proceso de formación de lo que es ser femenino y masculino. La niña aprende a ser obediente, pasiva, dependiente y subordinada al hombre mientras que al niño se le enseña a ser independiente, agresivo, fuerte y racional. Aún más, Silva Bonilla y otras (1990) plantean que la autorrealización de las mujeres en la esfera familiar está predicada sobre bases ideológicascognoscitivas, valorativas y prácticas que implican la pasividad y el sometimiento.

La familia reproduce lo que constituye la feminidad y la masculinidad en la vida cotidiana, reforzándola con cuentos y juguetes infantiles. Cuentos como «Blanca Nieves» y la «Bella Durmiente» en que las protagonistas son despertadas de sus sueños por un hombre que las salva casándose con ellas, perpetúan ideas de la mujer como débil y cuya máxima aspiración es el matrimonio. Existen muchos cuentos con ésta temática y otros en que se destacan la valentía, fuerza y sabiduría como atributos de los hombres mientras que la belleza, bondad y afectividad pertenecen a las mujeres. Además, padres y madres refuerzan estos atributos, regalándoles a las niñas juguetes que promueven el desarrollo de actitudes maternales orientadas al hogar y la familia, mientras que en los niños no sólo se fomentan destrezas motoras y mentales, sino conducta agresiva y violenta.

Otras instituciones como el sistema educativo, los medios masivos de comunicación y la religión también juegan un papel impor- 
tante en el proceso de socialización. Estas instituciones también contribuyen a la formación de estereotipos sobre los roles sexuales, es decir, creencias rígidas aplicadas para la generalidad de hombres y mujeres, asumiendo que los mismos son universales y naturales.

La escuela elemental o primaria, usualmente obligatoria en América Latina, comprende niños y niñas entre las edades de seis a doce años. Estas edades constituyen un terreno fértil para la asimilación de conductas y dentro de éstas de «roles» sexuales. Educadoras y educadores refuerzan y reprimen en forma distinta las actitudes y comentarios de niños(as). A los varones se les permite una mayor expresión agresiva y muscular, mientras que a las niñas se les acepta una mayor emotividad y sensibilidad a la crítica (González Suárez, 1990). Los textos escolares contribuyen a la formación de estereotipos, condicionando a los (las) estudiantes a pensar que la mujer es del hogar, responsable de las tareas domésticas y del cuidado de los hijos(as), y cuando trabaja fuera del hogar lo hace en ciertas profesiones tradicionalmente femeninas como maestras, secretarias, enfermeras y trabajadoras sociales. El examen sistemático de textos escolares de educación primaria ha ofrecido la oportunidad de conocer prejuicios contra la mujer en el lenguaje, imágenes, presentación de héroes y en general en el papel que se le asigna a la mujer en la sociedad (González Suárez, 1990; Picó, 1979).

Los medios masivos de comunicación ejercen una influencia fundamental en el proceso de transmisión ideológica. Tanto la televisión, como la radio y la comunicación escrita presentan estereotipos de la mujer como son el de ama de casa cuya máxima aspiración es la felicidad de su familia y como mujer joven y atractiva quien se convierte en objeto sexual. Cuando presentan a una mujer que trabaja por un salario, esta actividad es secundaria. Ella será capaz de abandonar el trabajo por el hombre y si conserva el empleo, su conducta será sumisa y dependiente con respecto a su jefe, quien obviamente es un hombre (Alegría, 1987). La situación es aún más degradante para la mujer cuando se examina la pornografía del cine y la televisión. La pornografía no sólo devalúa la imagen de la mujer como objeto sexual que puede ser explotada y manipulada sexualmente, sino que relaciona al sexo con alguna forma de violencia. Por ejemplo, presenta la violación en forma placentera para la mujer. Los programas especiales para niños y niñas presentan superhombres y monstruos buenos y malos, y la violencia extrema como la solución de los conflictos. Generalmente el niño va 
aprendiendo a ejercer la violencia para lograr sus propósitos y la niña a recibirla y a someterse a la misma.

La otra institución que juega un papel importante en el proceso de socialización es la iglesia. La enseñanza del perdón a las personas que nos ofenden, puede conducir a la aceptación de la violencia y a guardar silencio en las situaciones en que ocurre la misma. El ideal de la virgen María también se traduce en la búsqueda de la pureza, conduciendo a la consideración de la sexualidad como algo impuro, cuyo goce es permitido sólo a los hombres y que debe ser aceptado por la mujer como parte de las obligaciones del matrimonio (González Suárez, 1990).

Toda esta instrucción diferenciada de los sexos ejerce una influencia directa en los mismos. La niña va aprendiendo a ser sumisa, obediente y a imitar modelos como el de la Virgen María; mientras que el niño, a su vez comenzará muy pronto a aprender a sentirse dueño de la mujer con el poder de controlarla y someterla a su obediencia. Todo hombre, aún el no violento, utiliza cierto tipo de presión a la mujer para obtener los beneficios y privilegios a los que entiende tiene derecho cuando el hombre entiende que la mujer no está cumpliendo su rol y por lo tanto él no está percibiendo "su privilegio»; entonces cree que tiene derecho a castigar de múltiples maneras a la mujer. Asimismo, la violencia se ha legitimado como mecanismo de control social para mantener el esquema de autoridad patriarcal que mantiene a las mujeres subordinadas y dependientes (Guzmán Stein, 1994). La cultura ha facilitado la violencia, en la que los hombres son socializados para ejercerla legítimamente y las mujeres para aceptarla como algo natural, como su destino manifiesto.

\section{Violencia en la relación de pareja y salud pública}

Organismos internacionales en el área de la salud como son la Organización Mundial de la Salud (OMS) y la Organización Panamericana de la Salud (OPS), han reconocido la violencia intra-familiar como un problema de salud pública. Ambos organismos conceptúan la violencia como toda acción u omisión que en forma intencional y dirigida, ocasiona daño o lesión, física, mental, sexual o social. En 1992, el Programa de Mujer, Salud y Desarrollo en conjunto con los Ministerios de Salud de Centroamérica realizaron la primera reunión centroamericana sobre la violencia contra la mujer 
como un problema de salud pública (Subcomité sobre la Mujer, la Salud y el Desarrollo, 1999). Los estudios presentados en esa reunión revelaron, entre otros hallazgos, que la violencia hacia la mujer en la familia es reveladora de la inequidades de género; y que su invisibilidad tiene repercusiones en la salud de la mujer y en la calidad de la atención de los servicios. Más adelante, en 1996, la Asamblea Mundial de la OMS declaró la violencia como una prioridad de salud pública e instó a los países miembros a iniciar actividades para la prevención de la violencia y para atender sus efectos en la salud (Ellsberg, et al. 1998).

Como problema de salud pública, la violencia puede tener varias dimensiones y alcances. La dimensión física puede implicar el aislamiento de grupos vulnerables, especialmente mujeres, niñas y ancianas; que podrían impedir la promoción y prevención de la salud con consecuencias como la discapacidad y la muerte (Ver Anejo 1- Consecuencias de la violencia en la salud de las mujeres). Campbell y Soeken (1999) plantean que debido a que muchas mujeres maltratadas informan pérdida del conocimiento que no ha sido atendida, los dolores de cabeza crónicos, que muchas veces ellas describen, pueden constituir un diagnóstico inadecuado de la secuela de daño neurológico del maltrato. Aún más, los problemas no diagnosticados de audición, visión y concentración informados por mujeres maltratadas sugieren posibles problemas neurológicos de las heridas o lesiones (Cambell y Soeken, 1999).

La dimensión mental puede incluir estrés, trastornos de alimentación, desórdenes post-traumáticos, trastornos de personalidad, depresión y hasta el suicidio (Ver Anejo 1). En esta dimensión, también se ha incluido el comportamiento sexual de alto riesgo, incluyendo el Síndrome de Inmunodeficiencia Adquirida (SIDA). Los casos de mujeres infectadas y enfermas de SIDA en el mundo han aumentado alcanzando el $42 \%$ de los casos, y de las 7,000 nuevas infecciones que ocurren diariamente, el 50 por ciento corresponden a mujeres (ISIS Internacional, 2000). Aunque muchos de estos casos corresponden a la violencia en general que existe hacia la mujer y no solamente a la violencia en la relación de pareja, estas estadísticas ponen en evidencia las condiciones de desigualdad social y económica en que viven la mayor parte de ellas. Además, el abuso sexual se asocia con el alto riesgo a contraer otras enfermedades transmitidas sexualmente, a desgarre vaginal y anal, a infecciones de la vejiga, a disfunción sexual, dolor en la pelvis, infeccio- 
nes de orina, embarazos no atendidos, y otros problemas de salud relacionados con los genitales, que han sido documentados con mujeres maltratadas en albergues y escenarios de cuidado de la salud (Cambell \& Soeken, 1999).

En una investigación realizada en Nicaragua, se encontró que las mujeres maltratadas tenían seis veces más probabilidades de sufrir trastornos emocionales que las mujeres que nunca habían vivido violencia (Ellsberg et al.,1998, ver Anejo 2 - Violencia doméstica y trastornos emocionales). El equipo que condujo esta investigación aclaró que aunque no tenían información sobre el estado emocional de la mujer antes de recibir violencia física, la relación estadística tan fuerte indica que los trastornos emocionales deben ser considerados como un resultado y no como una causa de la victimización (Ellsberg et al., 1998). Asimismo, investigaciones en Estados Unidos han encontrado que la mujer maltratada, cuando se le compara con la que no lo es, tiene cuatro o cinco veces más probabilidades de requerir tratamiento psiquiátrico, y cinco veces más probabilidades de intentos de suicidio. Por otro lado, un estudio sobre los años de vida saludable perdidos por mujeres de 15 a 44 años demostró que la violencia de género representó una pérdida de 9.5 años de vida (ver Anejo 3 - La carga de la salud global...).

Las personas que laboran en el escenario de salud, son en muchas ocasiones las que primero atienden a las mujeres víctimas/ sobrevivientes de la violencia en la relación de pareja, y las que pueden ayudar a esta mujeres a tomar decisiones sobre su vida. Las mujeres pueden acudir a hospitales o centros de salud para recibir atención por alguna herida o golpe; como también en la atención prenatal o control de crecimiento y desarrollo de sus hijas e hijos, y esta es una gran oportunidad para detectar o indagar si está viviendo en violencia. En otras palabras, se podría decir que el sector salud es el único escenario al que acuden en algún momento las mujeres víctimas sobrevivientes de violencia en la relación de pareja. Sin embargo, este personal no puede promocionar la salud de estas mujeres, si no está capacitado para detectar o reconocer esta violencia, y si los hospitales o centros de salud no tienen un protocolo en el que se pregunte si ha existido o existe la violencia en la relación de pareja.

Ellsberg, et al. (1998) presenta algunas propuestas que incluyen: la atención a posibles síntomas y signos de violencia; preguntar a las usuarias acerca de sus experiencias de violencia como 
parte de la historia clínica de rutina; registrar los casos de abuso en el expediente médico; mantener una actitud de respeto y la confidencialidad de la información de las usuarias y los expedientes; y dar orientación y referir a servicios disponibles. Es importante recordar que ninguna propuesta será exitosa si no se modifican las ideas erróneas en torno a la relación de pareja. Entre las ideas más comunes encontramos que este tipo de violencia es un problema social o legal y no uno de salud pública, que es un problema personal y privado; y la creencia de que esta violencia no ocurre entre mujeres profesionales o en familias de mejor nivel económico.

\section{Prevención y atención de la violencia en la relación de pareja}

En la prevención y atención de la violencia en la relación de pareja es primordial el enfoque de trabajo multi-disciplinario, multi-institucional y multi-sectorial. Mis reflexiones sobre el tema parten de las experiencias de capacitación, docencia e investigación en Puerto Rico, Estados Unidos y algunos países centroamericanos. En primera instancia, se requiere el desarrollo de instrumentos que permitan la identificación apropiada de situaciones en las que ha ocurrido algún tipo de violencia en la relación de pareja. En Estados Unidos se han desarrollado normas y protocolos en hospitales para detectar esta violencia y ya se está evaluando la efectividad de algunos de esos procedimientos. En 1999, el Subcomité sobre la Mujer, la Salud y el Desarrollo de la OPS acordó establecer normas y protocolos en unidades de salud de Centroamérica (Subcomité sobre la Mujer, la Salud y el Desarrollo, 1999). Es urgente que estos procedimientos sean establecidos en todos los hospitales y centros de salud, y que su personal sea adiestrado para la utilización de estos procedimientos. Se puede comenzar en la formación de los profesionales de la salud, por ejemplo, en las escuelas de medicina y enfermería.

En segundo lugar, las discusiones en equipo de situaciones donde ha ocurrido la violencia en la relación de pareja por profesionales de la medicina, enfermería, trabajo social, psicología y educación, son muy acertadas en el análisis integral del problema. Tercero, las instituciones de salud deben trabajar en colaboración con otras instituciones como son las que le brindan servicios a la mujer y éstas a su vez tienen que trabajar con las instituciones de justicia. Estas 
instituciones pueden desarrollar una Red de Prevención y Atención en la que unan esfuerzos para desarrollar actividades como por ejemplo, la promoción de estilos de vida saludables y en el desarrollo de materiales educativos en un lenguaje sencillo. Por último, y de gran importancia, todas estas instituciones y profesionales tienen que considerar a otros sectores como son los grupos de apoyo a nivel voluntario o en organizaciones comunitarias, para poder trabajar hacia la paz familiar.

Cuando intervenimos con mujeres que han vivido violencia en la relación de pareja es importante, irrespectivamente de los escenarios de salud, de justicia o de servicios sociales, recordar o conocer lo siguiente:

\section{La manifestación del ciclo de la violencia.}

2. El muro del silencio alrededor de la violencia en la relación de pareja. Las mujeres prefieren mantenerse calladas cuando están expuestas a la violencia porque se trata del padre de sus hijos o hijas o del hombre que aman.

3. No responsabilizar a la mujer, tanto si permanece en esa relación como si decide poner fin a la misma.

4. Muchas mujeres se sienten culpables si no ayudan al agresor, pero se les debe ayudar a entender que su vida es demasiado valiosa para arriesgarla tratando de ayudar a quien está lastimándola brutalmente. Así mismo, es importante que la víctima sobreviviente entienda que un agresor aprende a usar la violencia como un modo de expresar coraje o frustración mucho antes que encuentre a su esposa. Esto no quiere decir que el hombre no necesita ayuda, sino que la mujer víctima/sobreviviente no es la más indicada para hacerlo.

5. Es necesario que los hombres que necesiten ayuda psicológica la obtengan, pero también deben participar de grupos de reeducación y discusión donde tengan la oportunidad de asumir responsabilidad por sus actos y adiestrase en todo lo que conlleva la verdadera democracia dentro de la familia.

6. La violencia se reproduce entre los niños y niñas que la observan pasivamente y entre aquellos(as) con impacto directo. 
Entre las alternativas que se han llevado a cabo y continúan vigentes está la construcción o restauración de albergues o refugios para mujeres agredidas con sus hijos e hijas. No obstante, estos albergues no son la solución aislada al problema de violencia doméstica. Debe trabajarse con las mujeres, lo cual podría ser en consejería o grupos de discusión y con los niños(as) que presencian ésta violencia. Es imprescindible evaluar el contexto socio-cultural para determinar la necesidad de un refugio y contar con la protección legal, de forma tal que la mujer que tiene que salir del hogar con sus hijos(as) no pierda sus derechos.

Es necesario desarrollar estrategias que eduquen a los medios de comunicación y a las instituciones públicas y que sensibilicen al público en general, incluyendo a las mujeres mismas, sobre las desastrosas consecuencias personales y nacionales de la violencia continua sobre la mujer (Carrillo, 1995). Además, es necesario, entre otras cosas, que los funcionarios en la administración de justicia (policías, fiscales, jueces, etc.) sean adiestrados en el manejo del problema y sean sensibles a las necesidades de las víctimas y de sus hijos(as); que el personal médico cuente con protocolos claramente establecidos para auxiliar a las víctimas; que el sistema educativo incluya cursos sobre la violencia doméstica y ofrezca servicios a los estudiantes que viven las mismas.

Debemos ser creativos y creativas en la búsqueda de alternativas. Los hombres no agresivos deben involucrarse y servir de modelos a los que de una u otra forma ejercen violencia, para que aprendan a lidiar con el conflicto a través del diálogo o de otras formas no violentas. Las mujeres tenemos que continuar participando en la defensa de los derechos adquiridos para que se implanten satisfactoriamente y continuar luchando por aquellos que aún no hemos logrado. La profesión de trabajo social, compuesta en su mayoría por mujeres, tiene la responsabilidad de asumir un rol más prominente en la identificación de violencia en todas las situaciones que interviene y participar en la educación para la paz en el hogar. Es imprescindible también incidir en todas las instituciones socializadoras: la familia, el sistema educativo, la iglesia, los medios masivos de comunicación, para que los valores y normas que se enseñen estén dirigidos a la paz y al respeto de la humanidad. Finalmente, todos y todas tenemos que dirigir nuestro esfuerzo hacia la erradicación de aquellas desigualdades y concepciones estereotipadas de la mujer que promueven la violencia. 


\section{Referencias}

Alegría, Idsa. E. (1987). La representación de la mujer trabajadora en la televisión en Puerto Rico. HOMINES-Revista de Ciencias Sociales, Universidad Interamericana de Puerto Rico, 4: 289-293.

Burgos Ortiz, Nilsa M. (1993, 22-26 febrero). Reflexiones en torno al proceso de aprobación y defensa de legislación sobre la violencia contra la mujer en Puerto Rico. Ponencia presentada en el V Congreso Internacional e Interdisciplinario de la Mujer, San José, Costa Rica.

Burgos Ortiz, Nilsa M., Sharratt, Sara y Trejos, Leda (1988). La mujer en Latinoamérica: Perspectivas sociales y psicológicas. Buenos Aires: Editorial HVMANITAS.

Campbell, Jacquelyn C. \& Soeken, Karen L. (1999). Women's responses to battering over time: An analysis of change. Journal of Interpersonal Violence, 14 (1), 21-40.

Carrillo, Roxana (1995). La violencia contra la mujer: Un obstáculo para el desarrollo. En Charlotte Bunch y Roxana Carrillo, violencia de género: Un problema de desarrollo y derechos humanos. New Jersey: Center for Women's Leadership.

Cervantes Islas, Francisco (2000). Desafíos masculinos para frenar la violencia hacia las mujeres. Modemmujer-Red de comunicación electrónica. México: Internet.

Colectivo Ideologías y Vivencias de los Géneros (1995). La más casera de las violencias sociales: Violencia contra la pareja. Puerto Rico: Editorial De Una Orilla a Otra Orilla.

Ellsberg, Mary; Arcas, Carmen C.; Montenegro, Tania; Norori, Lorna y Quintanilla, Margarita (1998). ¿Cómo atender a las mujeres que viven situaciones de violencia doméstica? Managua, Nicaragua: Red de Mujeres Contra la Violencia, y Departamento de Medicina Preventiva y Salud Pública de la Escuela de Medicina UNAN-León. 
Ellsberg, Mary; Peña, Rodolfo; Herrera, Andrés; Liljestrand; y Winkvist (1998). Confites en el infierno: Prevalencia y características de la violencia conyugal hacia las mujeres en Nicaragua. Managua/León, Nicaragua: Red de Mujeres Contra la Violencia, Departamento de Medicina Preventiva, UNAN-León y Departamento de Epidemiología y Salud Pública, Umea University, Suecia..

Estado Libre Asociado de Puerto Rico (1989). Ley para la prevención e intervención con la violencia doméstica, Ley \#54. Puerto Rico: Autor.

González Suárez, M. (1990). El sexismo en la educación. Costa Rica: Editorial de la Universidad de Costa Rica.

Guzmán Stein, Laura (1994). La paz y los derechos humanos en las vidas de las mujeres latinoamericanas. Visión y prospectiva del desarrollo social. Barranquillas, Colombia: VIII Congreso Nacional de Trabajo Social.

ISIS Internacional (2000). SIDA una expresión de violencia de género. Modemmujer. México: Internet.

Maldonado, Mare Ann (1990). Violencia contra la mujer por ser mujer: Mitos y estereotipos. Puerto Rico Health Sciences Journal, 9 (1): 111-116.

Pico, Isabel (1979). Machismo y educación. Puerto Rico: Comisión para el Mejoramiento de los Derechos de la Mujer.

Silva Bonilla, Ruth M. (1985). El marco de la violencia contra las mujeres en la vida conyugal. Río Piedras: Centro de Investigaciones Sociales, CERES.

Silva Bonilla, Ruth M.; Rodríguez, Jeannette; Cáceres, Virgen; Martínez, Lourdes; y Torres, Nilsa (1990). Hay amores que matan: La violencia contra la mujer en la vida conyugal. Río Piedras: Ediciones Huracán, Inc. 
Subcomité sobre la Mujer, la Salud y el Desarrollo (1999). Abordaje del problema de la violencia contra la mujer en la política de reforma del sector de la salud en Centroamérica. Washington, DC: OPS/OMS.

Torres Hernández, Nilsa I. (1999). Hombres, masculinidades y violencia doméstica: Percepciones de algunos hombres sobre los actos de maltrato que se cometen contra la pareja. Disertación doctoral, Departamento de Psicología, UPR, Río Piedras.

Valle Ferrer, Diana (1998). Estrategias de enfrentamiento de las mujeres contra la violencia. En Diana Valle Ferrer, Lillian Albite Vélez e Islia Rosado López, Violencia en la familia: Una perspectiva crítica. Puerto Rico: Ediciones de Familia y Comunidad.

Walker, Leonor (1980). The battered women. New York: Harper and Row Publishers. 


\section{Anejo 1}

\section{CONSECUENCIAS DE LA VIOLENCIA EN LA SALUD DE LAS MUJERES}

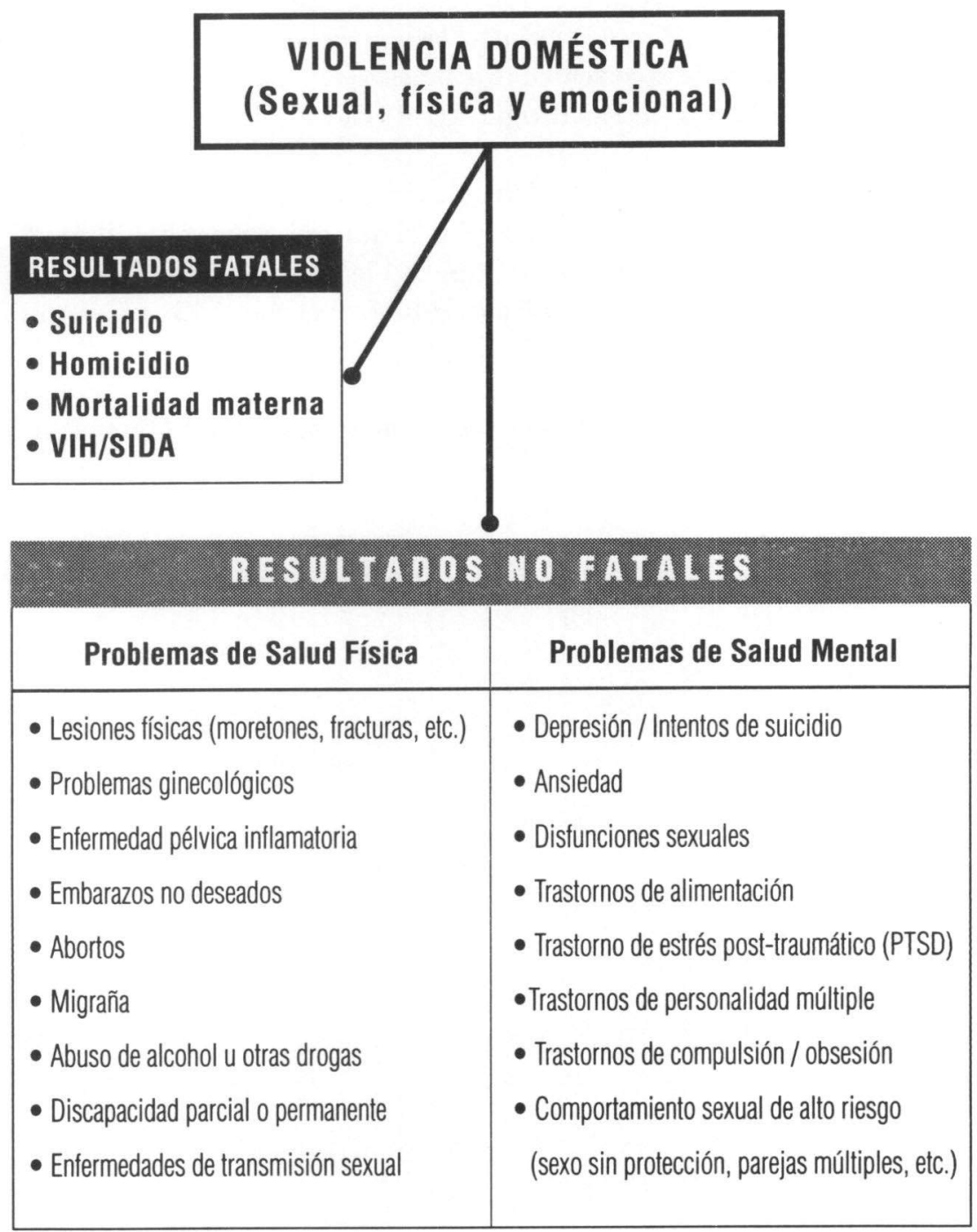

Adaptado de Ellsberg, et al. (1998). Confites en el infierno, 2da. ed. Nicaragua: Red nacional de Mujeres Contra la Violencia. Departamento de Medicina Preventiva, UNANLeón y Departamento de Epidemiología y Salud Pública, Umea University, Suecia. 


\section{VIOLENCIA DOMÉSTICA Y PROBLEMAS EMOCIONALES EN LAS MUJERES $(n=360)$}

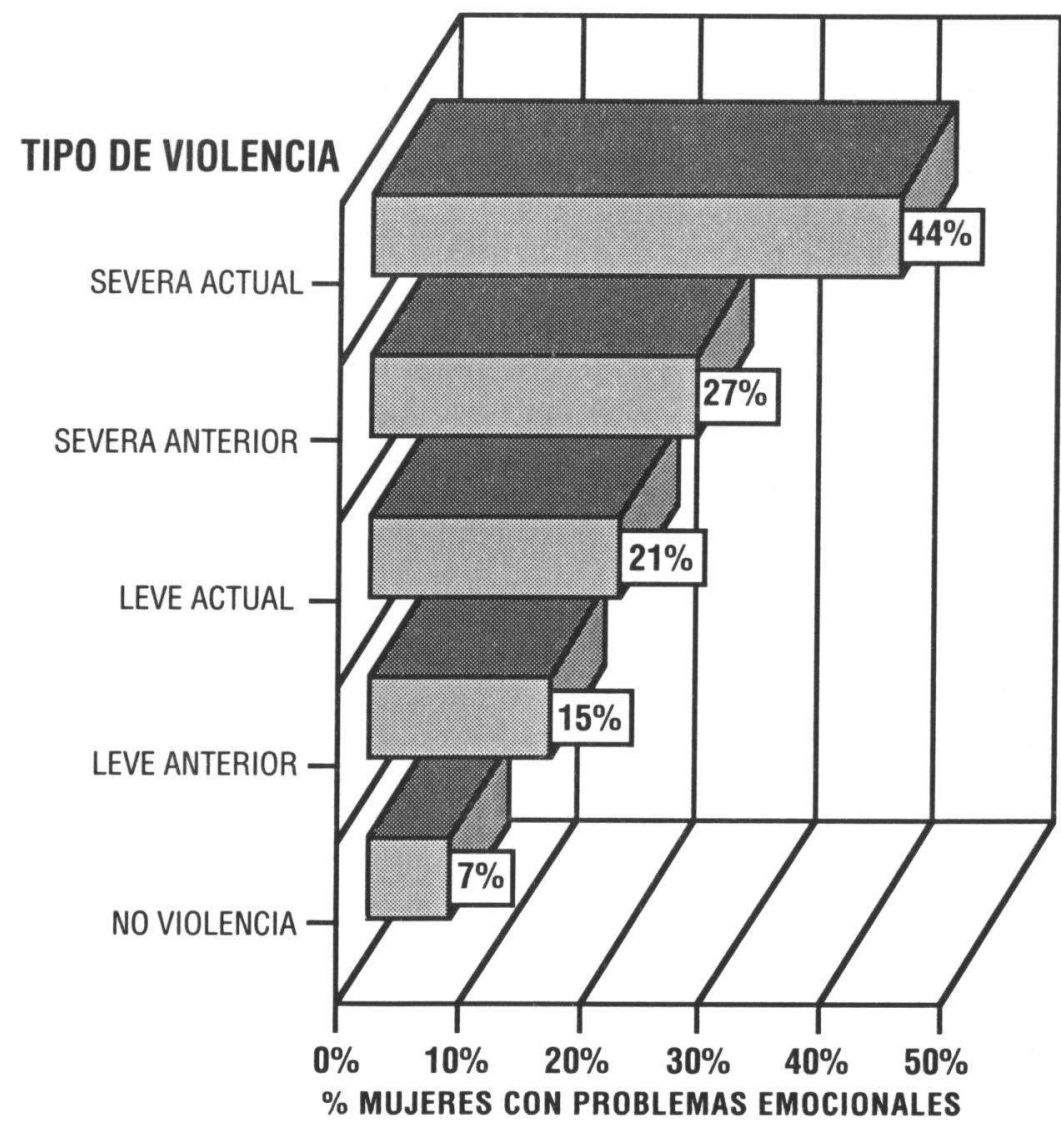

En esta figura se presenta la prevalencia de problemas emocionales en las mujeres según la severidad de la violencia y el momento en que se dio.

Tomado de Ellsberg, et al. (1998). Confites en el infierno, 2da. ed. Nicaragua: Red Nacional de Mujeres Contra la Violencia. Departamento de Medicina Preventiva, UNANLeón y Departamento de Epidemiología y Salud Pública, Umea University, Suecia. 


\section{LA CARGA DE SALUD GLOBAL ESTIMADA POR CONDICIONES SELECCIONADAS EN MUJERES DE 15 A 44 AÑOS DE EDAD}

CONDICIÓN

AÑOS DE VIDA SALUDABLES

PERDIDOS AJUSTADOS

POR DISCAPACIDAD

Condiciones maternas

29.0

- Sepsis

10.0

- Parto Obstruido

Enfermedades de Transmisión Sexual

(excluyendo a VIH/SIDA)

Inflamación pélvica

Tuberculosis

$\mathrm{VIH} / \mathrm{SIDA}$

Enfermedad cardiovascular

10.5

Violación y violencia doméstica

Todos los cánceres

- Mamas

- Cuello uterino

Accidentes automovilísticos

Guerra

Malaria 